\title{
Activatable clinical fluorophore- quencher antibody pairs as dual molecular probes for the enhanced specificity of image-guided surgery
}

Girgis Obaid

Bryan Q. Spring

Shazia Bano

Tayyaba Hasan 


\title{
Activatable clinical fluorophore-quencher antibody pairs as dual molecular probes for the enhanced specificity of image-guided surgery
}

\author{
Girgis Obaid,,$^{a, \dagger}$ Bryan Q. Spring, ${ }^{\mathrm{b}, \dagger}$ Shazia Bano, ${ }^{\mathrm{a}}$ and Tayyaba Hasan ${ }^{\mathrm{a}, \mathrm{c}, \star}$ \\ ${ }^{a}$ Massachusetts General Hospital and Harvard Medical School, Wellman Center for Photomedicine, Boston, Massachusetts, United States \\ ${ }^{b}$ Northeastern University, Department of Physics, Boston, Massachusetts, United States \\ 'Harvard University and Massachusetts Institute of Technology, Division of Health Sciences and Technology, Cambridge, Massachusetts, \\ United States
}

\begin{abstract}
The emergence of fluorescently labeled therapeutic antibodies has given rise to molecular probes for image-guided surgery. However, the extraneous interstitial presence of an unbound and nonspecifically accumulated probe gives rise to false-positive detection of tumor tissue and margins. Thus, the concept of tumor-cell activation of smart probes provides a potentially superior mechanism of delineating tumor margins as well as small tumor deposits. The combination of molecular targeting with intracellular activation circumvents the presence of extracellular, nonspecific signals of targeted probe accumulation. Here, we present a demonstration of the clinical antibodies cetuximab (cet, anti-EGFR mAb) and trastuzumab (trast, anti-HER-2 mAb) conjugated to Alexa Fluor molecules and IRDye QC-1 quencher optimized at the ratio of 1:2:6 to provide the greatest degree of proteolytic fluorescence activation, synonymous with intracellular lysosomal degradation. The cet-AF-Q-C1 conjugate $(1: 2: 6)$ provides up to 9.8 -fold proteolytic fluorescence activation. By preparing a spectrally distinct, irrelevant sham IgG-AF-QC-1 conjugate, a dual-activatable probe approach is shown to enhance the specificity of imaging within an orthotopic AsPC-1 pancreatic cancer xenograft model. The dual-activatable approach warrants expedited clinical translation to improve the specificity of image-guided surgery by spectrally decomposing specific from nonspecific probe accumulation, binding, and internalization. ๑ 2017 Society of Photo-Optical Instrumentation Engineers (SPIE) [DOI: 10.1117/1.JBO.22.12.121607]
\end{abstract}

Keywords: molecular imaging; image-guided surgery; fluorescence-guided resection; clinical antibodies; targeted optical probes. Paper 170299SSLR received May 6, 2017; accepted for publication Aug. 1, 2017; published online Aug. $29,2017$.

\section{Introduction}

Since the seminal report of the in-human safety and tumorspecificity of fluorescent antibodies in work described by Rosenthal et al., leveraging FDA-approved therapeutic antibodies as fluorescent clinical molecular probes for image-guided surgery is becoming an ever-growing reality. ${ }^{1-4}$ Rosenthal et al. fabricated a fluorescent conjugate of IRDye $800 \mathrm{CW}$ with the human-murine chimeric monoclonal antibody cetuximab for the in vivo clinical imaging of epidermal growth factor receptor (EGFR) in head and neck cancer patients. ${ }^{1}$ This development in the clinical imaging of cancer using targeted, optically active biologics has been warranted by the long-standing unmet clinical need for assistance in surgical navigation. ${ }^{4}$ Prior attempts to address this critical need were initially approached in 1948 by administering nonspecific fluorescein dye to image perfused brain tumors in patients. ${ }^{5}$ More recently, far-red and near-infrared dyes, namely methylene blue (MB) and indocyanine green (ICG), exhibiting superior suitability for imaging deep tissue have proven to be valuable fluorescence contrast agents for the mapping of sentinel lymph nodes, monitoring blood perfusion, and imaging vascular and lymphatic pathologies to assist surgical procedures. ${ }^{6,7}$ In the context of oncology, limited reports have demonstrated the detection of hepatic and breast

\footnotetext{
*Address all correspondence to: Tayyaba Hasan, E-mail: thasan @ mgh.harvard .edu

${ }^{\dagger}$ Both authors contributed equally
}

malignancies using intravenously administered ICG, leveraging delayed dye interstitial clearance from tumors to provide selectivity. ${ }^{8,9}$ However, the weak selectivity and absence of discrete tumor specificity of fluorophores, such as ICG and MB, render them unreliable for accurate tumor detection and image-guided surgery. The necessity for increased tumor specificity has since lead to strategies that leverage the tumor tissue's inherent capacity to synthesize an accumulated amount of fluorescent protoporphyrin IX following the exogenous administration of 5-aminolevulinic acid. ${ }^{10}$ In June 2017, 5-aminolevulinic acid received FDA approval as Gleolan ${ }^{\circledR}$ for image-guided surgery of glioma and a number of clinical trials also leveraging the approach to guide surgical resection are ongoging. ${ }^{4}$ The described strategies, although powerful in their own right, provide weak specificity when imaging cancer and can provide heterogeneous intratumoral signals, requiring secondary approaches to improve their specificity and homogeneity of labeling. ${ }^{11,12}$ The earliest demonstration of leveraging fluorescent antibodies for imaging human disease tissue was reported by Folli et al. who intravenously administered fluorescent anticarcinoembryonic antigen antibodies to patients with primary colorectal carcinoma and imaged the tissue ex vivo following surgical resection. ${ }^{13}$ The first demonstration of in-human molecular imaging in patients was reported in 2011 by van Dam et al. who visualized ovarian cancer metastases, of which $90 \%$ to $95 \%$ overexpress the folate receptor- $\alpha$, using a

$1083-3668 / 2017 / \$ 25.00$ @ 2017 SPIE 
folate-fluorescein conjugate to guide radical cytoreductive surgery. ${ }^{14}$ Following the first in-human demonstration of image-guided surgery using cetuximab-IRDye $800 \mathrm{CW}$ in $2015,{ }^{1}$ a phase I trial was initiated for the use of the conjugate for image-guided surgery of head and neck cancer, (NCT01987375). A second trial imaging EGFR using cetuximab-IRDye $800 \mathrm{CW}$ is also pending. Four phase I, clinical trials using an IRDye $800 \mathrm{CW}$ conjugate of the antivascular endothelial growth factor receptor antibody have also been initiated to image familial adenomatous polyposis (NCT01691391), breast cancer (NCT01508572), rectal cancer (NCT01972373), and premalignant esophageal lesions (NCT02129933). ${ }^{4}$ An additional pending clinical trial will also leverage an antiprostate-specific membrane antigen antibody to image prostate cancer (NCT02048150). Of particular relevance to this study, a current clinical trial is performing intraoperative imaging of pancreatic cancer using a cetuximab-IRDye $800 \mathrm{CW}$ conjugate (NCT02736578), further motivating our demonstration here of a dual-activateable probe approach in an orthotopic model of pancreatic ductal adenocarcinoma.

Building on the wealth of antibody-based molecular probes for image-guided surgery, the concept of proteolytic probe activation is an elegant means to enhance the specificity of protein-based molecular probes. We have previously reported an activatable photosensitizer-cetuximab conjugate whereby proteolytic intracellular degradation of the antibody conjugate resulted in tumor-specific activation of imaging and photodynamic therapy. ${ }^{15-18}$ These antibody probes have been demonstrated to be effective therapeutic agents with the potential to serve a dual diagnostic and therapeutic function. ${ }^{15,19-21}$ Furthermore, they can be leveraged to mop up the surgical bed from residual, undetectable microscopic disease. ${ }^{15,20}$ Static quenching of fluorophores when confined in proximity to one another on an antibody can exist through self-quenching or by the introduction of a Förster resonance energy transfer- and contact-based dark quencher. ${ }^{22-26}$ In this approach, activation is dependent on the spatial separation of statically quenched fluorophores upon lysosomal degradation. In this study, we present a flexible platform optimized for the activation of far-red and near-infrared antibody fluorophore conjugates using IRDye QC-1 as a broad-spectrum quencher to enable the synthesis of molecular-targeted, activatable fluorescent probes over a spectrum of fluorophore colors, given that sufficient spectral overlap exists between the fluorophore's emission and the quencher's absorption profile.

As described in the methods section, varying ratios of Alexa Fluor 700 (AF700) and IRDye QC-1 (QC-1) were conjugated to cetuximab to obtain a final dye/antibody ratio of 8 [Fig. 1(a)] to provide conjugates with increasing degrees of the dark quencher QC-1 [Fig. 1(b)]. This dye ratio was chosen to avoid over labeling that compromises the biological activity and specificity of the antibody. The conjugates were then subject to a simulation of intracellular lysosomal proteolysis using trypsin in vitro
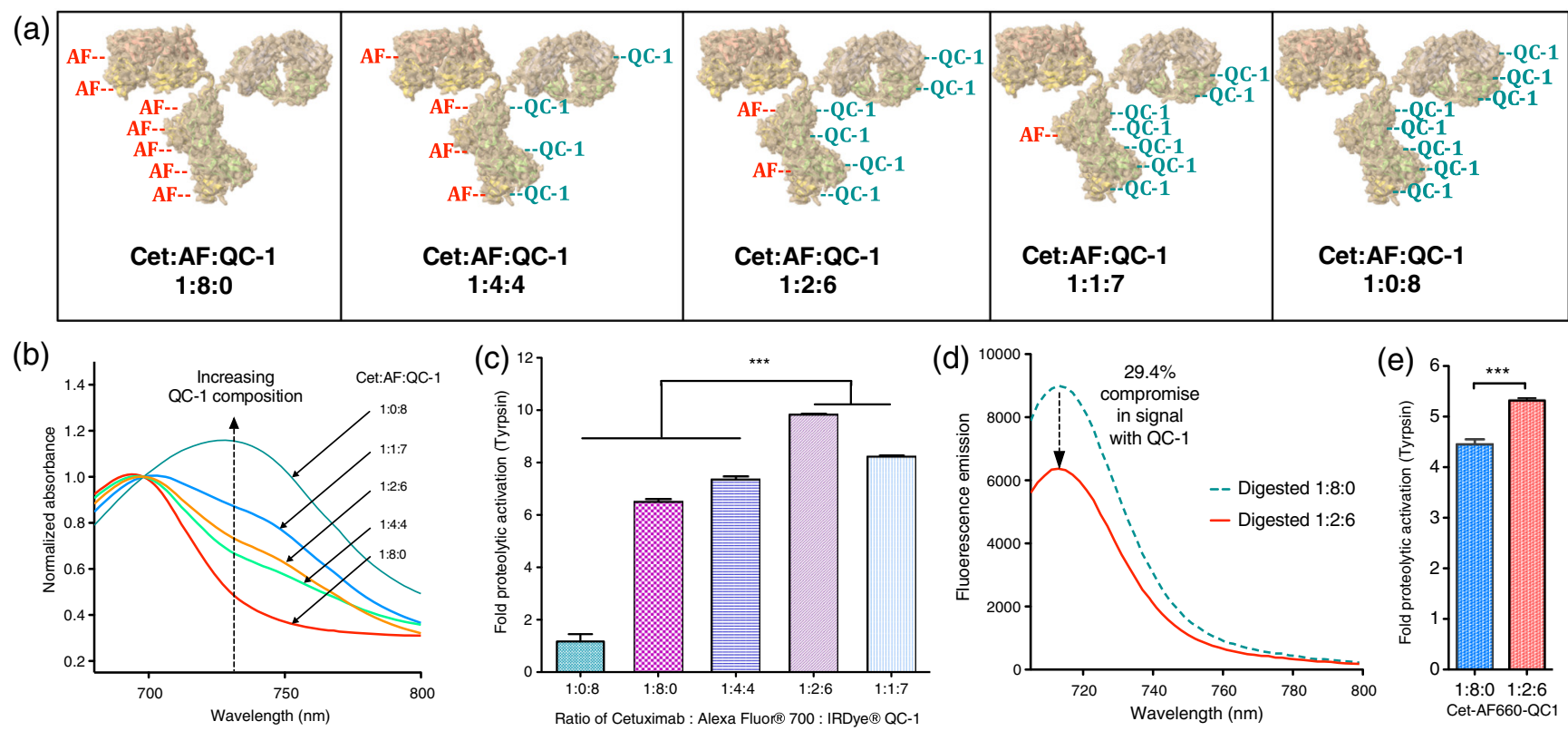

Fig. 1 (a) Schematic representation of the panel of cetuximab (cet) conjugated to varying ratios of Alexa Fluor 700 (AF) and IRDye QC-1 (QC-1) amounting to a total of eight chromophores per antibody. (b) Normalized UV-visible absorption spectra of the varied ratio cetuximab conjugates with increasing degrees of QC-1 incorporation. (c) Proteolytic activation of the panel of varying fluorophore-quencher ratio cetuximab conjugates by $24 \mathrm{~h}$ of trypsin digestion revealed that 1:2:6 ratio of cetuximab:Alexa Fluor 700:IRDye QC-1 exhibited the highest degree of activation with a 9.8-fold increase in fluorescence following probe digestion. (d) Raw fluorescence emission spectra of digested, activated cetuximab:Alexa Fluor 700 with and without IRDye QC-1 demonstrate that the increased specificity provided by the quencher results in only $29.4 \%$ compromise in brightness. (e) Activation was also demonstrated in Alexa Fluor 660 cetuximab conjugates showing an improvement in fold activation with QC-1 incorporation at a ratio of 1:2:6. Data points are mean \pm S.D., statistical significance was calculated using a oneway ANOVA analysis with Tukey post-test comparison in (c) and using a two-tailed $t$-test in (e). Cetuximab is represented by the refined structure of an intact IgG2a monoclonal antibody (Protein Data Bank; PDB ID: $1 \mathrm{IGT})^{27}$ and the three-dimensional (3-D) structure was projected using Jmol: an open-source Java viewer for chemical structures in $3-D .^{28}$ 
to assess the degree of activation the probes exhibited. With increasing QC-1 composition, the degree of cetuximab activation, reaching a maximum of $\sim 10$-fold activation with the optimal 1:2:6 ratio of cet:AF:QC-1 [Fig. 1(c)]. Further incorporation of $\mathrm{QC}-1$ at a 1:1:7 ratio of cet:AF:QC-1 resulted in an inferior degree of activation compared to $1: 2: 6$. The enhanced specificity provided by the QC- 1 in the cet-AF700 conjugates also results in a $29.4 \%$ compromise in signal, as shown by the raw fluorescence emission spectra [Fig. 1(d)], which is not anticipated to be problematic in the clinic, as probe specificity rather than fluorophore brightness remains the major unmet clinical need. ${ }^{1}$ Enhanced activation is also demonstrated by the incorporation of the QC-1 in cetuximab AF660 conjugates [Fig. 1(e)], exemplifying the flexibility of this approach to synthesize activatable conjugates using other fluorophores.

Fundamental principles of the static quenching and intracellular activation approach are shown schematically [Fig. 2(a)] along with basic spectroscopic data showing the overlap of the QC-1 absorption spectrum with the fluorescence emission of the exemplary far-red AF dye pre- and postactivation for the optimal $1: 2: 6$ ratio [Fig. 2(b)].

To further test that the optical ratio identified for cetuximabbased probes holds for other antibodies, we compared the efficiency of self-quenching ( $1: 8: 0$; no QC-1 dark quencher) with the $1: 2: 6$ ratio for trastuzumab [Fig. 3(a)] and an IgG isotype control [Fig. 3(b)]. The enhanced activation potential using the QC-1 dark quencher at the optimal ratio held for all three of the

(a)
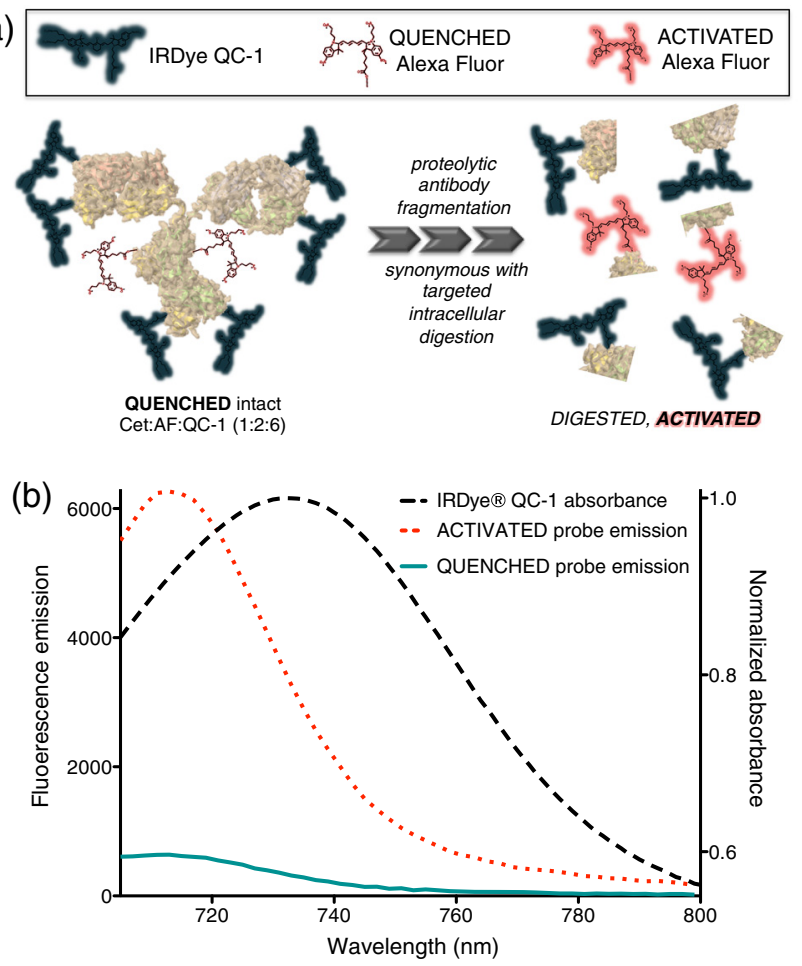

Fig. 2 (a) Schematic representation of the targeted intracellular proteolysis of optimal cetuximab-Alexa Fluor 700-IRdye QC-1 (1:2:6) leading to the spatial separation of the fluorophore from the quencher and subsequent fluorescence activation (the structure of the proprietary Alexa Fluor 700 is depicted by the structure of the related dye Alexa Fluor 647). (b) Fluorescence emission spectra of quenched, intact cet:AF:QC-1 $(1: 2: 6)$ and the activated, proteolyzed cet:AF:QC-1 $(1: 2: 6)$ with respect to the absorption spectrum of the IRDye QC-1 quencher. antibody conjugates [Fig. 3(c)]. This finding suggests that this approach can be applied to a wide range of fluorescent dyes to potentially construct a panel of multicolor probes (i.e., any dye that overlaps with the broad QC-1 absorption spectrum, which extends from $\sim 500$ to $900 \mathrm{~nm})^{24}$ for efficient quenching of visible, far-red, and near-infrared dyes).

We then performed a preliminary xenograft study in orthotopic pancreatic cancer (AsPC-1) to demonstrate tumor-specific activation of dual-activatable tracers (Fig. 4). Fluorescence image-guided surgery was simulated by acquiring hyperspectral near-infrared fluorescence images of dual-activatable cetuximab-AF660-QC-1 (1:2:6) and IgG-AF700-QC-1 (1:2:6) sham probes coinjected intravenously into mice bearing the orthotopic AsPC-1 pancreatic cancer xenografts. The tumor margin is clearly visualized by the cetuximab-based activatable probe whereas the nonspecific IgG-activatable probe enabled visualization of nonspecific accumulation with significant background in the pancreas and surrounding organs [Figs. 4(a)-4(j)]. Figure 4(j) is a heat map (using the "fire" look up table, LUT, in ImageJ) image for 0 to 5 that quantitatively shows specific binding where cet is above the IgG nonspecific level, thus delineating the tumor margins more accurately, as compared to the activateable cet alone in Fig. 4(i). The qualitative margin delineation presented here is preliminary and would require histological analysis for validation. The heat map was generated by subtraction of the image intensity histogram mode of each raw image (cet and $\mathrm{IgG}$ ) to set the mean background to zero. Each image is then normalized (divided) by its overall mean signal intensity. For each pixel, the ratio cet/IgG - 1 was calculated, such that $0(1-1)$ indicates no specific binding and values greater than 0 highlight specific binding, and this is presented as the heat map image in Fig. 4(j). The ratio used for image analysis of the dual-activateable probe technique was adapted from a previously reported study. ${ }^{29}$

Future work will exploit this new activatable dual tracer approach to perform quantitative imaging of cellular uptake and molecular target levels in the tumor, extending a new powerful approach to perform dual tracer molecular fluorescence imaging that enables quantitative imaging of receptor binding and cellular uptake based on pharmacokinetic modeling. ${ }^{30,31}$

Finally, an exciting area of future development will able to integrate this multicolor, activatable imaging probe platform with theranostic agents that in addition to fluorescence also produce cytotoxic-reactive species (e.g., singlet oxygen) to perform therapy ${ }^{15,20}$ (i.e., tumor-targeted, activatable photoimmunotherapy, and taPIT). This development will have potential to enable surgeons to selectively "mop up" residual microscopic disease left behind in the surgical bed.

\section{Materials and Methods}

\subsection{Antibody Chromophore Conjugates}

Cetuximab (Erbitux ${ }^{\circledR}$; Ely Lily), trastuzumab (Herceptin ${ }^{\circledR}$; Genentech), and human IgG isotype control (Pierce) were prepared to $2 \mathrm{mg} / \mathrm{ml}$ concentrations in bicarbonate solution $(0.1 \mathrm{M}$, $\mathrm{pH}$ 8.0). Antibody solutions in $500 \mu \mathrm{l}$ aliquots were added to Alexa Fluor ${ }^{\circledR} 700$ or 660 NHS esters $(10 \mathrm{mg} / \mathrm{ml}$ in anhydrous DMSO; Invitrogen) premixed with or without IRDye ${ }^{\circledR}$ QC-1 NHS ester $(10 \mathrm{mg} / \mathrm{ml}$ in anhydrous DMSO; LiCor) at a molar excess of antibody that provides eight dye molecules per antibody, given that Alexa Fluor ${ }^{\circledR}$ NHS ester reactions were found to be $53.7 \%$ efficient and IRDye ${ }^{\circledR}$ QC-1 NHS ester reactions were 
(a)

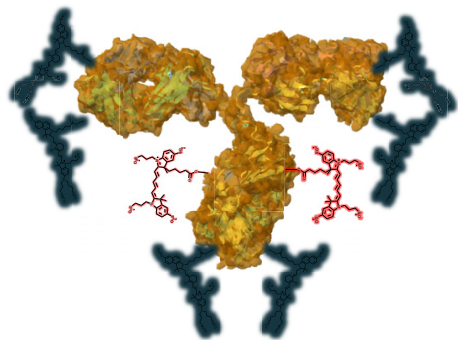

trastuzumab : AF : QC-1 $(1: 2: 6)$

(b)

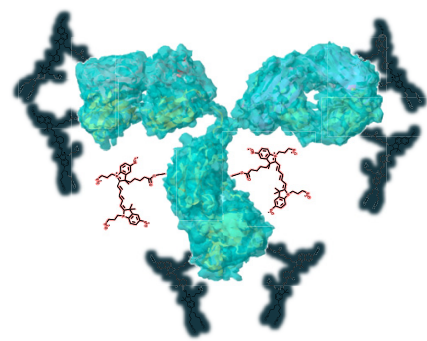

IgG isotype control : AF : QC-1 $(1: 2: 6)$

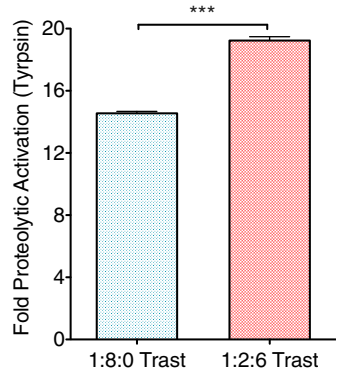

Ratio of Antibody : Alexa Fluor ${ }^{\circledR} 700$ : IRDye ${ }^{\circledR}$ QC-1

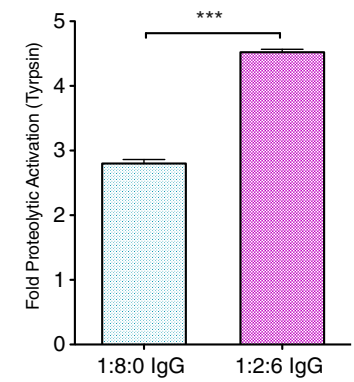

Ratio of Antibody : Alexa Fluor ${ }^{\circledR} 700$ : IRDye ${ }^{\circledR}$ QC-1

(c)

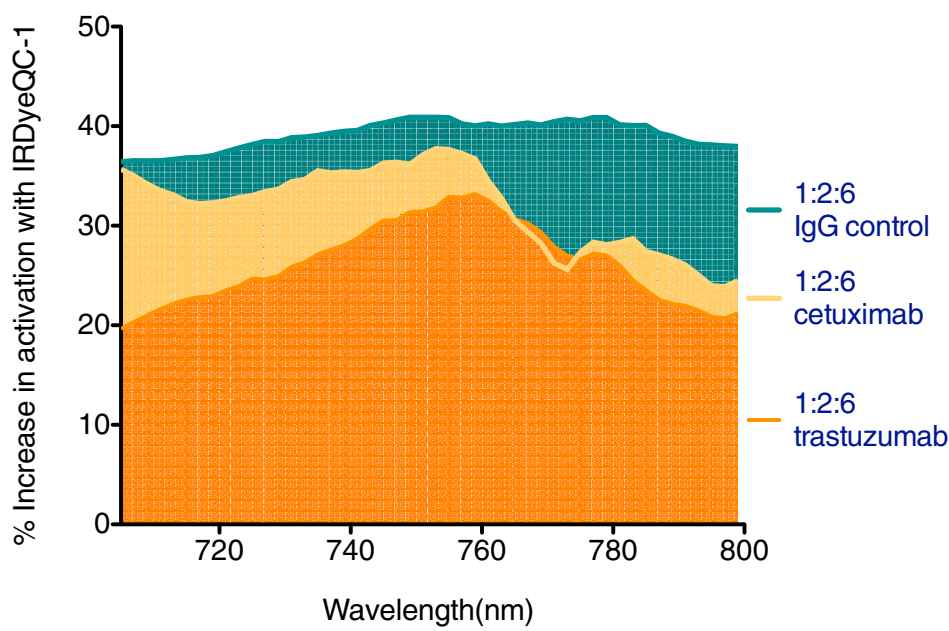

Fig. 3 Schematic representations and degree of proteolytic activation of (a) trastuzumab and (b) IgG isotype control conjugated to the optimal Ab:AF:QC-1 ratio of 1:2:6. (c) Percentage increases in activation over the entire emission spectrum of Alexa Fluor 700 by introducing IRDyeQC-1 at the optimal ratio. Data points are mean \pm S.D. and statistical significance was calculated using a one-way ANOVA analysis with Tukey post-test comparison. Cetuximab, trastuzumab, and human IgG isotype control are represented by the refined structure of an intact IgG2a monoclonal antibody (PDB ID 1 IGT) ${ }^{27}$ and the 3-D structure was projected using Jmol: an open-source Java viewer for chemical structures in 3-D. ${ }^{28}$

found to be $63.1 \%$ efficient at $4 \mathrm{~h}$ room temperature conjugation reactions. To provide antibody:Alexa Fluor $^{\circledR}$ : IRDye ${ }^{\circledR}$ QC-1 ratios of $1: 8: 0,1: 4: 4,1: 2: 6,1: 1: 7$, and $1: 0: 8$, antibodies were reacted with Alexa Fluor ${ }^{\circledR}$ NHS esters and IRDye ${ }^{\circledR}$ QC1 NHS esters at respective ratios of $1: 14.9: 0,1: 7.4: 6.3$, $1: 3.7: 9.5,1: 1.9: 11.1$, and $1: 0: 12.7$ for $4 \mathrm{~h}$ at room temperature. Following conjugation, the antibody chromophore conjugates were separated from unconjugated dye by size exclusion chromatography using Thermo Fisher Scientific Alexa Fluor antibody conjugate separation resin equilibrated with $1 \times$ PBS. Dye concentrations were measured using UV-visible spectrophotometry dilutions in DMSO $\left(\mathrm{AF} 660 \varepsilon_{668 \mathrm{~nm}}=132,000 \mathrm{M}^{-1} \mathrm{~cm}^{-1}\right.$; AF700 $\varepsilon_{702 \mathrm{~nm}}=205,000 \mathrm{M}^{-1} \mathrm{~cm}^{-1} ;$ and $\mathrm{QC}-1 \varepsilon_{788 \mathrm{~nm}}=$ $\left.98,000 \mathrm{M}^{-1} \mathrm{~cm}^{-1}\right)$. Antibody concentrations were calculated using the Pierce ${ }^{\mathrm{TM}}$ BCA Protein Assay Kit (Thermo Fisher Scientific) and standard curves of respective antibody solutions in PBS. Antibody-chromophore ratios were determined by the molar ratio of antibody to chromophores following purification.

\subsection{Trypsin Simulation of Intracellular Proteolysis}

Antibody-chromophore conjugates were incubated in the presence of $1 \%$ trypsin for $24 \mathrm{~h}$ at $37^{\circ} \mathrm{C}$ in triplicate. Fluorescence spectra of the Alexa Fluor dye were measured with and without trypsinization to determine the degree of quenching and activation. Changes in fluorescence activation were measured using integrated emission spectra of the preoteolytically cleaved 

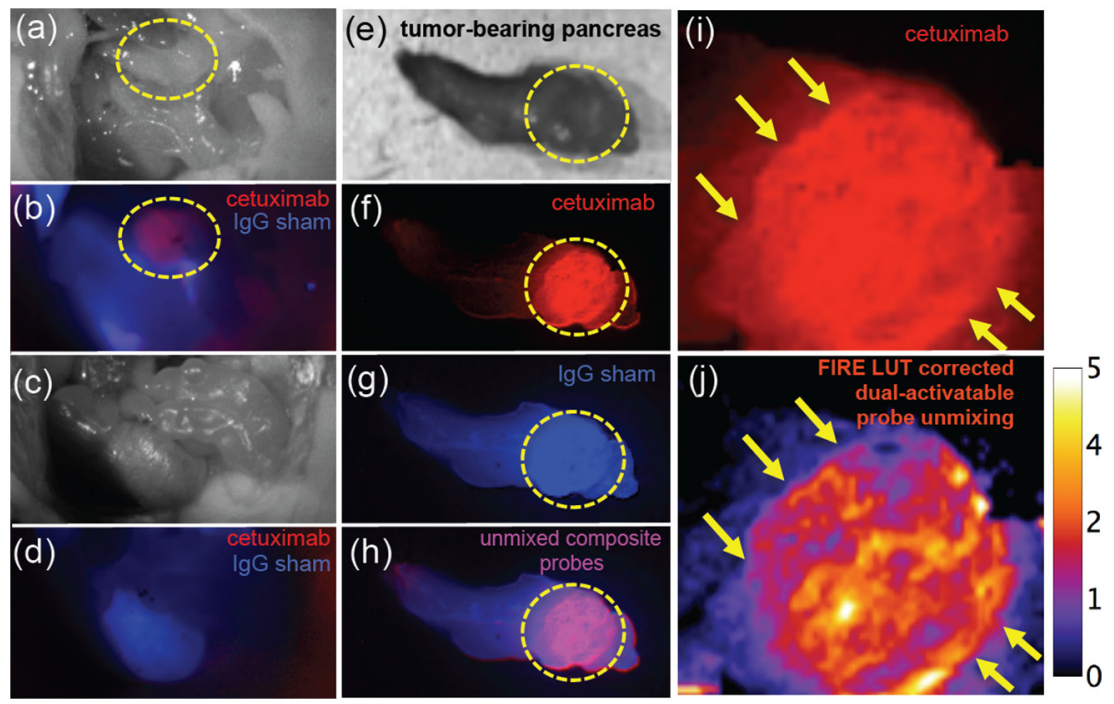

Fig. 4 Hyperspectral near-infrared fluorescence imaging a murine model of orthotopic AsPC-1 pancreatic cancer xenografts simulating fluorescence image-guided surgery using the dual-activatable cetuximab-AF660-QC-1 (1:2:6, red) and IgG-AF700-QC-1 (1:2:6, blue) sham probes. (a) White light and (b) hyperspectral composite images of the abdominal cavity and the pancreas bearing an orthotopic ASPC-1 tumor 15 days after implantation. (c) Following resection of the tumor-bearing pancreas (white light), (d) no evidence of residual disease remains as shown in the hyperspectral composite image. (e) Following resection of the AsPC-1 tumor-bearing pancreas, tumor margins are undetected by white light, (f) whereas unmixed cetuximab-AF660-QC-1 (1:2:6, red) enables tumor visualization, (g) unmixed IgG-AF700-QC-1 (1:2:6, blue) enables visualization of nonspecific antibody binding profiles, and $(h)$ the composite hyperspectral image of the pancreas identifies areas where nonspecific binding contributes to the signal of the targeted probe. (i) Tumor margins delineated by activatable cetuximab-AF660-QC-1 (1:2:6, red) . A heat map image (using the "fire" LUT in ImageJ) subtracting the nonspecific component of cet binding provides superior specificity of imaging and an improved tumor margin delineation are shown by yellow arrows in (j) (see text for image computation details).

antibody normalized to the integrated emission spectra of the intact antibody chromophore conjugate.

\subsection{Orthotopic Pancreatic Cancer In Vivo Simulation of Image-Guided Surgery}

A left abdominal flank incision was made in male 6- to 8-week old Swiss nude mice to exteriorize the pancreas. The body of the pancreas was injected with AsPC- 1 cancer cells $\left(1 \times 10^{6}\right.$ cells in $50 \mu \mathrm{l}$ of $50 \%$ matrigel) orthotopically implanted. The incision was sutured and the tumors were left to develop for 14 days. A cocktail of cet:AF660:QC-1- and IgG:AF700:QC-1-activatable probes $(10 \mathrm{mg} / \mathrm{kg}$ each) was injected into the tail vein $24 \mathrm{~h}$ prior to imaging. The abdominal flank was then reopened and the mice were imaged hyperspectrally using the Maestro whole mouse imaging system (Perkin Elmer) before and after removal of the pancreas. Baseline spectra of free AF660 and AF700 in PBS were also measured using the Maestro. The mouse and tumor images were hyperspectrally unmixed using the defined basis spectra at $1 \mathrm{~nm}$ resolution and were used to generate unmixed composite images of specific (cet:AF660: QC-1) and nonspecific (IgG:AF700:QC-1) probe binding.

\section{Disclosures}

Drs. Girgis Obaid, Shazia Bano, Bryan Spring, and Tayyaba Hasan have no conflicts of interest, financial or otherwise.

\section{Acknowledgments}

The support of the National Institutes of Health Grant R01CA160998 and P01CA084203 to T. H., K22CA181611 to B.Q.S. and financial support by the Bullock-Wellman postdoctoral fellowship to G. O are gratefully acknowledged. The technical assistance and insightful discussions with Dr. Zhiming Mai and Dr. Akilan Palanisma are also gratefully acknowledged. The animal procedure for the injection of antibody fluorophore conjugates was approved by the Institutional Animal Care and Use Committee (IACUC). No human subjects were involved in this study.

\section{References}

1. E. L. Rosenthal et al., "Safety and tumor specificity of cetuximabIRDye800 for surgical navigation in head and neck cancer," Clin. Cancer Res. 21(16), 3658-3666 (2015).

2. E. L. Rosenthal et al., "The status of contemporary image-guided modalities in oncologic surgery," Ann. Surg. 261(1), 46-55 (2015).

3. Q. T. Nguyen and R. Y. Tsien, "Fluorescence-guided surgery with live molecular navigation: a new cutting edge," Nat. Rev. Cancer 13(9), 653-662 (2013).

4. E. L. Rosenthal et al., "Successful translation of fluorescence navigation during oncologic surgery: a consensus report," J. Nucl. Med. 57(1), 144-150 (2016).

5. G. E. Moore et al., "The clinical use of fluorescein in neurosurgery: the localization of brain tumors," J. Neurosurg. 5(4), 392-398 (1948).

6. S. L. Troyan et al., "The FLARE intraoperative near-infrared fluorescence imaging system: a first-in-human clinical trial in breast cancer sentinel lymph node mapping," Ann. Surg. Oncol. 16(10), 29432952 (2009).

7. M. V. Marshall et al., "Near-infrared fluorescence imaging in humans with indocyanine green: a review and update," Open Surg. Oncol. J. 2(2), 12-25 (2010).

8. B. Alacam et al., "Pharmacokinetic-rate images of indocyanine green for breast tumors using near-infrared optical methods," Phys. Med. Biol. 53(4), 837-859 (2008). 
9. T. Ishizawa, Y. Bandai, and N. Kokudo, "Fluorescent cholangiography using indocyanine green for laparoscopic cholecystectomy: an initial experience," Arch. Surg. 144(4), 381-382 (2009).

10. W. Stummer et al., "Fluorescence-guided surgery with 5-aminolevulinic acid for resection of malignant glioma: a randomised controlled multicentre phase III trial," Lancet Oncol. 7(5), 392-401 (2006).

11. B. Ortel et al., "Differentiation enhances aminolevulinic acid-dependent photodynamic treatment of LNCaP prostate cancer cells," Br. J. Cancer 87(11), 1321-1327 (2002).

12. S. Anand et al., "Low-dose methotrexate enhances aminolevulinatebased photodynamic therapy in skin carcinoma cells in vitro and in vivo," Clin. Cancer Res. 15(10), 3333-3343 (2009).

13. S. Folli et al., "Immunophotodiagnosis of colon carcinomas in patients injected with fluoresceinated chimeric antibodies against carcinoembryonic antigen," Proc. Natl. Acad. Sci. U. S. A. 89(17), 7973-7977 (1992).

14. G. M. van Dam et al., "Intraoperative tumor-specific fluorescence imaging in ovarian cancer by folate receptor-alpha targeting: first inhuman results," Nat. Med. 17(10), 1315-1319 (2011).

15. B. Q. Spring et al., "Selective treatment and monitoring of disseminated cancer micrometastases in vivo using dual-function, activatable immunoconjugates," Proc. Natl. Acad. Sci. U. S. A. 111(10), E933-E942 (2014).

16. M. D. Savellano and T. Hasan, "Photochemical targeting of epidermal growth factor receptor: a mechanistic study," Clin. Cancer Res. 11(4), 1658-1668 (2005).

17. M. D. Savellano and T. Hasan, "Targeting cells that overexpress the epidermal growth factor receptor with polyethylene glycolated BPD verteporfin photosensitizer immunoconjugates," Photochem. Photobiol. 77(4), 431-439 (2003).

18. N. S. Soukos et al., "Epidermal growth factor receptor-targeted immunophotodiagnosis and photoimmunotherapy of oral precancer in vivo," Cancer Res. 61(11), 4490-4496 (2001).

19. D. Mew et al., "Photoimmunotherapy: treatment of animal tumors with tumor-specific monoclonal antibody-hematoporphyrin conjugates," J. Immunol. 130(3), 1473-1477 (1983).

20. L. S. Moore et al., "Photoimmunotherapy of residual disease after incomplete surgical resection in head and neck cancer models," Cancer Med. 5(7), 1526-1534 (2016).

21. M. Mitsunaga et al., "Cancer cell-selective in vivo near infrared photoimmunotherapy targeting specific membrane molecules," Nat. Med. 17(12), 1685-1691 (2011).

22. R. Weissleder et al., "In vivo imaging of tumors with protease-activated near-infrared fluorescent probes," Nat. Biotechnol. 17(4), 375-378 (1999).

23. S. A. Marras, F. R. Kramer, and S. Tyagi, "Efficiencies of fluorescence resonance energy transfer and contact-mediated quenching in oligonucleotide probes," Nucleic Acids Res. 30(21), 122e (2002).

24. X. Peng et al., "A nonfluorescent, broad-range quencher dye for Forster resonance energy transfer assays," Anal. Biochem. 388(2), 220-228 (2009).

25. J. F. Lovell et al., "Activatable photosensitizers for imaging and therapy," Chem. Rev. 110(5), 2839-2857 (2010).
26. H. Kobayashi and P. L. Choyke, "Target-cancer-cell-specific activatable fluorescence imaging probes: rational design and in vivo applications," Acc. Chem. Res. 44(2), 83-90 (2011).

27. L. J. Harris et al., "Refined structure of an intact IgG2a monoclonal antibody," Biochemistry 36(7), 1581-1597 (1997).

28. http://www.jmol.org/ (April 2017)

29. J. T. Liu et al., "Quantifying cell-surface biomarker expression in thick tissues with ratiometric three-dimensional microscopy," Biophys. J. 96(6), 2405-2414 (2009).

30. S. C. Davis et al., "Dynamic dual-tracer MRI-guided fluorescence tomography to quantify receptor density in vivo," Proc. Natl. Acad. Sci. U. S. A. 110(22), 9025-9030 (2013).

31. K. M. Tichauer et al., "Microscopic lymph node tumor burden quantified by macroscopic dual-tracer molecular imaging," Nat. Med. 20(11), 1348-1353 (2014).

Girgis Obaid is a postdoctoral research fellow at the Wellman Center for Photomedicine, Massachusetts General Hospital and Harvard Medical School. His doctoral thesis under the guidance of Prof. David Russell (School of Chemistry, University of East Anglia, U. K.) explored glycan-selective bioconjugated gold nanoparticles as targeted photodynamic therapy (PDT) platforms. His postdoctoral fellowship explores antibody-targeted nanotechnologies for the selective imaging and PDT-based combination regimens under the mentorship of Tayyaba Hasan.

Bryan Q. Spring is an assistant professor of biomedical physics (Northeastern University, Boston). His doctoral work with Robert M. Clegg (Center for Biophysics and Quantitative Biology, University of Illinois at Urbana-Champaign) focused on developing fluorescence lifetime and quantitative FRET imaging. He developed patent pending technology for molecular imaging and selective treatment of cancer micrometastases during his postdoctoral fellowship in Tayyaba Hasan's laboratory (Wellman Center for Photomedicine, Massachusetts General Hospital and Harvard Medical School).

Shazia Bano obtained her doctorate degree in physics (medical) from The Islamia University of Bahawalpur, Pakistan, under the guidance of Prof. Dr. Muhammad Afzal Khan. She is currently a visiting scholar at the Wellman Center for Photomedicine, Massachusetts General Hospital and Harvard Medical School, working on the fabrication of targeted nanoconstructs for near infrared imaging of cancer and image-guided anticancer phototherapies.

Tayyaba Hasan is a professor of dermatology at Harvard Medical School and a professor of health sciences and technology (Harvard-MIT). The focus of her research is on image-guided PDT of cancer and infections with over 200 publications. She is an inventor of Visudyne $®$ for the PDT treatment of age-related macular degeneration. She also leads NCl-funded international Programs on developing image guided PDT of oral cancer, skin cancer and pancreatic cancer. 